\title{
Vigilância do câncer relacionado ao trabalho: sobre as Diretrizes 2012 publicadas pelo INCA
}

\author{
Surveillance of work-related cancer: on the 2012 Guide \\ published by INCA (Brazilian National Cancer Institute)
} ${ }^{1}$ Professor Titular do Departamento de
Epidemiologia da Faculdade de Saúde
Pública da Universidade de São Paulo.
Membro do Conselho Editorial da RBSO.
Em 30 de abril de 2012 foi lançada, no Instituto Nacional de Câncer José de Alencar Gomes da Silva (INCA), a publicação Diretrizes para a vigilância do câncer relacionado ao trabalho (INSTITUTO NACIONAL DO CÂNCER JOSÉ DE ALENCAR GOMES DA SILVA, 2012), que amplia e aprofunda outra anterior do próprio INCA: Vigilância do câncer relacionado ao trabalho e ao ambiente, com edições em 2006 e 2010. A iniciativa é bem-vinda, necessária e de pronta utilidade para os que operam na linha de frente das ações de proteção à saúde do trabalhador, uma vez que os tumores malignos representam atualmente a segunda causa de morte no mundo, conforme consta no próprio texto. As neoplasias relacionadas ao trabalho têm grande potencial de prevenção, visto que, distintamente de outros fatores de risco para câncer, como o tabagismo, o controle das exposições cancerígenas ocupacionais é menos dependente de decisões individuais e pode ser garantido pela vigilância e pelas intervenções nos ambientes de trabalho.

Os capítulos 1 a 3 das Diretrizes tratam das questões relativas ao câncer decorrente de exposições ocupacionais. No capítulo 4, discutem-se as políticas públicas e os direitos do trabalhador com câncer. Os capítulos 5 a 9 são operacionais e delineiam diferentes estratégias de sistemas de vigilância. No capítulo 10 são discutidas as aplicações e as limitações dos estudos epidemiológicos.

Na introdução das Diretrizes, é apresentado um panorama geral e breve acerca da fração atribuível à ocupação na ocorrência de câncer nas populações, o que tem sido objeto de estudos detalhados. Há grande incerteza em relação a quase todos os dados destas estimativas. Doll e Peto (1981) avaliaram que os fatores ocupacionais seriam responsáveis por $4 \%$ das mortes por câncer na população americana, com variação de incerteza entre $2 \%$ e $8 \%$. Quanto ao câncer de pulmão, cuja relação causal com a ocupação é mais evidente, a contribuição dos fatores ocupacionais chegaria a $15 \%$ das mortes nos homens e a $5 \%$ nas mulheres. Já no século XXI, Nurminen e Karjalainen (2001) estimaram que metade das mortes relacionadas ao trabalho na Finlândia seria decorrente de câncer, o de pulmão representando $54 \%$ das mortes por todos os tumores malignos associados a exposições ocupacionais. Rushton, Hutchings e Brown (2008), no Reino Unido, calcularam as frações atribuíveis à ocupação para seis tipos de câncer: pulmão, bexiga, cavidades sinonasais, leucemias, mesotelioma e pele não melanoma. Concluíram 
que 4,9\% desses tumores (homens 8\%; mulheres 1,5\%) seriam decorrentes de exposições a cancerígenos nos locais de trabalho. Nestes dois últimos estudos, as frações atribuíveis às exposições ocupacionais no câncer de pulmão ultrapassaram $20 \%$.

Em 2009, o total de 113.801 benefícios de auxílio doença por câncer foi concedido pela Previdência Social e apenas $751(0,66 \%)$ foram registrados como tendo relação com a ocupação do trabalhador. Tome-se o exemplo do câncer de pulmão cujas taxas de incidência e mortalidade são próximas devido ao seu prognóstico reservado. Em 2010, foram registradas 21.779 mortes por câncer de pulmão no Brasil: 12,2\% das mortes por câncer. Assumindo-se como aplicáveis à população brasileira as frações atribuíveis estimadas para Finlândia e Reino Unido, pelo menos 4.355 destas mortes por câncer de pulmão foram decorrentes de exposições a cancerígenos nos locais de trabalho. Conclui-se que há grande subnotificação do câncer de origem ocupacional no país.

O documento das Diretrizes, em seu Quadro 19 (p. 71), relata apenas 19 pesquisas acerca de fatores de risco ocupacionais relativos ao câncer, publicadas entre 1991 e 2009 no Brasil, onde a vigilância do câncer relacionado ao trabalho é incipiente. Países com maior tradição de pesquisa nesta área possuem sistemas de vigilância sobre agentes cancerígenos nos locais de trabalho mais bem estruturados e, desde 1980, têm assinalado consideráveis reduções destas exposições (STRAIF, 2008).

A natureza multifatorial e complexa da etiologia do câncer torna, na maioria das vezes, difícil sua caracterização como de origem ocupacional. No capítulo 3 das Diretrizes assumiu-se que uma determinada ocupação (e possível exposição a algum agente) seria cancerígena com base nos resultados de estudos epidemiológicos. Entretanto, é importante ponderar que os estudos epidemiológicos de cunho observacional tão somente identificam associações, não definindo causalidade. Assim, afirmações feitas no capítulo 3 devem ser tomadas com cautela. Algumas das ocupações assinaladas como de risco para alguns tumores decorrem de associações obtidas em investigações epidemiológicas, porém sem consistência com resultados de outros estudos. Por exemplo, o trabalho exercido no setor elétrico e a consequente maior exposição aos campos magnéticos de muito baixa frequência foram relacionados aos tumores do sistema nervoso central, de mama, à leucemia e ao melanoma de pele. Todavia, os resultados dos estudos que exploraram o assunto são contraditórios e não permitem inferir explícita relação causal. A Agência Internacional de Pesquisa em Câncer (INTERNATIONAL AGENCY FOR RESEARCH ON CANCER, 2002), da Organização Mundial da Saúde, classifica os campos magnéticos de baixa frequência como possivelmente cancerígenos para os humanos (Grupo 2B) com base no seu possível efeito nas leucemias infantis, e não nas leucemias ou em outros tumores malignos em adultos.

Para fins de vigilância, é prudente manter o foco nos ramos de atividade e nas ocupações classificadas pela IARC como definitiva ou potencialmente cancerígenas, além, obviamente, dos agentes potencialmente cancerígenos para os humanos, listados, respectivamente, no Anexo 3 (p. 169) e no Anexo 2 (p. 161) das Diretrizes (INSTITUTO NACIONAL DO CÂNCER JOSÉ DE ALENCAR GOMES DA SILVA, 2012). Nos procedimentos de avaliação da IARC (INTERNATIONAL AGENCY FOR RESEARCH ON CANCER, 2006), o painel de especialistas para definir uma ocupação ou um agente como cancerígeno avalia a consistência dos resultados entre os estudos epidemiológicos, bem como de estudos em modelos animais e de mecanismos carcinogênicos.

O mérito principal das Diretrizes para a vigilância do câncer relacionado ao trabalho é de contribuir com os fundamentos estruturais para a vigilância das exposições ocupacionais a agentes cancerígenos no país. A carga do câncer é crescente na população brasileira, sendo importante ressaltar que as ações de vigilância desenvolvidas hoje somente terão efeito em longo prazo, pois a latência dos tumores malignos sólidos é da ordem de 10 a 50 anos, embora possa ser menor nas neoplasias hematopoiéticas.

A publicação das Diretrizes para a vigilância do câncer relacionado ao trabalho representa um marco não apenas para os que desenvolvem atividades de proteção à saúde dos trabalhadores nas instâncias do Sistema Único de Saúde, mas também para as políticas de prevenção do câncer na sociedade brasileira.

\section{Referências}

INSTITUTO NACIONAL DO CÂNCER JOSÉ DE ALENCAR GOMES DA SILVA. Coordenação Geral de Ações Estratégicas. Coordenação de Prevenção e Vigilância do Câncer Relacionado ao Trabalho e ao Ambiente. Diretrizes para a vigilância do câncer relacionado ao trabalho. Rio de Janeiro: INCA, 2012.
Disponível em: <http://www1.INCA.gov.br/INCA/Arquivos/ diretrizes_cancer_ocupa.pdf $>$. Acesso em: 28 maio 2012.

DOLL, R.; PETO, R. The causes of cancer: quantitative estimates of avoidable risks of cancer in the United States today. Journal of the National Cancer Institute, v. 66, n. 6, p. 1192-1308, 1981. 
NURMINEM, M.; KARJALAINEN, A. Epidemiologic estimate of the proportion of fatalities related to occupational factors in Finland. Scandinavian Journal of Work, Environment and Health, v. 27, n. 3, p. 161213, 2001.

RUSHTON, L.; HUTCHINGS, S.; BROWN, T. The burden of cancer at work: estimation as the first step to prevention. Occupational and Environmental Medicine, v. 65, n.12, p. 789-800, 2008.

STRAIF, K. The burden of occupational cancer (Editorial). Occupational and Environmental Medicine, v. 65, n. 12, p. 787-788, 2008.

\section{INTERNATIONAL AGENCY FOR RESEARCH ON}

CANCER. Non-ionizing radiation, Part 1: Static and extremely low-frequency (ELF) electric and magnetic fields. Lyon: IARC, 2002. (IARC Monographs on the evaluation of carcinogenic risk to humans. Vol. 80). Disponível em: <http://monographs.iarc.fr/ENG/Monographs/vol80/ mono80.pdf $>$. Acesso em: 08 jun. 2012.

. IARC Monographs on the evaluation of carcinogenic risk to humans: Preamble. Lyon: IARC, 2006. (Amended January 2006). Disponível em: <http://monographs.iarc.fr/ENG/Preamble/ CurrentPreamble.pdf>. Acesso em: 05 junho 2012. 